\title{
Control del poder: una historia de la evolución del concepto de separación de poderes
}

\section{Control of power: a history of the evolution of the concept of separation of powers}

\author{
María Andrea Enríquez Marín \\ Instituto de Investigaciones Jurídicas \\ Universidad Nacional Autónoma de México \\ Recibido: 10/09/13 \\ Aprobado: 03/11/2013 \\ andrea.enri88@gmail.com \\ «El poder tiende a corromper, \\ el poder absoluto corrompe absolutamente»
}

Lord Acton

\section{RESUMEN}

La separación de poderes es uno de los grandes mecanismos para lograr la limitación del poder buscado por el liberalismo político. Su objetivo de evitar el despotismo y garantizar la libertad de los individuos no ha cambiado con el paso de los años, pero la forma de entender al poder político y sus técnicas de organización sí, por lo que la teoría, estructura y manifestación de la separación de poderes también ha sufrido modificaciones, al grado de plantearse incluso su superación por autores como Karl Loewenstein. Tomando como guía la tesis de Michel Troper sobre la polisemia de este concepto, este trabajo explorará los seis distintos sentidos de la locución, nutriéndolos con los trabajos de algunos autores clásicos y contemporáneos, y sugiriendo que cada concepto en realidad representa una nueva etapa evolutiva. Posteriormente, el trabajo expone la teoría del control del poder -expuesta por el propio Loewenstein y Diego Valadés, entre otros autorescomo una alternativa 0 una conceptualización más acabada de estas ideas. Este siguiente paso evolutivo tiene implicaciones importantes debido a los constantes cambios institucionales y constitucionales de todos los países, ya que estas modificaciones no deben realizarse con el "enfoque del mejor sistema", sino para resolver los problemas específicos de cada contexto, siempre tomando en consideración que al final lo que importa en el Estado Constitucional de Derecho es lograr un efectivo control del poder.

\section{PALABRAS CLAVE}

Estado Constitucional de Derecho, Separación de poderes, Libertad individual, Monarquía.

\section{ABSTRACT}

The separation of powers is one of the large mechanisms for limiting the power provided by the political liberalism. It tries to avoid despotism and ensure the freedom of the individual, which has not changed over the years. The way to understand the political power and organizational techniques developed by theory, it has been modified, even to the extent of overcoming raised by authors like Karl Loewenstein. Guided by the theory of Michel Troper on the polysemy of this concept, this paper will explore six different senses of the phrase; sustain it with the works of some classic and contemporary authors, and suggesting that each concept actually represents a new evolutionary stage. The paper presents the theory of control of power-forward by 
Loewenstein and Diego Valadés- as an alternative or a more complete conceptualization of these ideas. This evolutionary step has important implications because of the constant institutional and constitutional changes in all countries. These changes should not be made with the "best system approach", but to solve the specific problems of each setting, always taking into consideration that in the end what matters in the constitutional rule of law is to achieve effective control of power.

\section{KEY WORDS}

Constitutional state, separation of powers, individual freedom, monarchy.

\section{INTRODUCCIÓN}

La separación de poderes es, después de los derechos, el segundo gran mecanismo de limitación del poder buscado por el liberalismo político. Esta protesta ideológica contra el absolutismo monolítico de la monarquía en los siglos XVII y XVIII (Loewenstein, 1976) en favor de la libertad individual fue formulada teóricamente por primera vez por Locke, pero fue Montesquieu quien propuso una formulación más elaborada de esta importante institución liberal (Salazar, 2006). El objeto no ha cambiado, ya que siempre se ha buscado evitar el despotismo y garantizar la libertad de los individuos, incluso de la misma mayoría ${ }^{1}$, pero la forma de entender al poder político y sus técnicas de organización han variado con el tiempo, de tal forma que cuando algunos autores hablan de separación del poder, otros lo distinguen de la división del poder, otros más de los checks and balances ("pesos y contrapesos") y autores como Loewenstein plantean la superación de la separación del poder, proponiendo una nueva división teórica de las funciones estatales en cuyo centro se encuentra el control del poder (1976: 54-72).

Tomando como guía la tesis de Michel Troper sobre la evolución histórica 0 polisemia del concepto de "separación de poderes", en este trabajo se esbozarán en primer lugar los seis distintos sentidos de la locución, y se señalará la relación entre estos conceptos y los sistemas presidencial y parlamentario, los cuales pueden ser considerados la expresión formal más evidente (Troper, 2006).
Posteriormente se proseguirá a exponer la teoría del control del poder como una alternativa 0 una conceptualización más acabada de estas ideas, una teoría que nos permite explicar fenómenos actuales de diseño institucional como la introducción de instituciones de corte parlamentario en regímenes presidenciales y viceversa en una búsqueda por mejorar los sistemas democráticos en el mundo. El objeto de esta propuesta es subrayar que éste no es un debate obsoleto, sino que es necesario para los actuales procesos de democratización y que debe ser tomado en consideración, que no se trata de "acercarse cada vez más al 'mejor sistema' ", y que se debe lidiar con los problemas específicos de las incipientes democracias tomando en consideración que su principal objetivo como democracias constitucionales es al final el control del poder.

Antes del análisis de los seis sentidos de "separación del poder" es importante aclarar tres cuestiones, las dos primeras acertadamente apuntadas por Riccardo Guastini (2000): en primer lugar, el vocablo "poder" condensa en sí dos significados, las funciones del Estado -entendidas como actividad o clase de actos- y los órganos del Estado que ejercitan estas funciones; la segunda cuestión, que se concluye de lo anteriormente expuesto, es que la separación de los poderes consistirá, por lo tanto, en una doble separación -la separación de las funciones y la separación de los órganos-; la tercera, que será más adelante profundizada, es que la interpretación más difundida en el siglo XX de la separación de poderes distingue dos principios de la misma, cuya preeminencia según el caso determinará en buena

1 "What can be done, while respecting the principle of majority rule, to safeguard the people against de consequences of their own ignorance or impetuosity?". Bryce, James, Modern Democracies, London, MacMillan and Co., Limited, vol. II, 1921, pág. 429 
medida la organización del poder en cada Estado: la especialización y la independencia.

\section{LOS SEIS SENTIDOS DE LA SEPARACIÓN DEL PODER}

\subsection{La separación del poder como una regla negativa}

Autores como Charles Eisenmann (1933) han sostenido en diversos trabajos desde hace ya muchos años que la interpretación de la obra el Espíritu de las Leyes de Montesquieu ha sido errónea, puesto que nunca habla de una separación de poderes en su obra, ni con esta denominación, ni con ninguna otra. Incluso en el modelo de la Constitución inglesa de la época, objeto de estudio y elogios en la obra, no se encuentran reglas de especialización 0 independencia, que son hoy los dos grandes principios de la separación de poderes. ¿Cuál era entonces la organización del poder a la que se referían tanto Montesquieu como sus contemporáneos?

Troper (1990) señala en su análisis histórico que la concepción del Poder Legislativo durante el siglo XVIII era distinta a la actual, en el sentido de que era considerado como el poder preeminente, al expresar la "voluntad general del Estado" (Montesquieu, 2007:147), mientras que la función ejecutiva era concebida como una función subordinada. En el Espíritu de las Leyes de Montesquieu (p.148-152) puede incluso verse esto con bastante claridad al hablar sobre las funciones de control entre los poderes, en que no son consideradas con la misma importancia, debido a la preeminencia de uno y la naturaleza limitada del otro:

Si el poder ejecutivo no tiene el derecho de contener los intentos del legislativo, éste será un poder despótico, porque pudiendo atribuirse toda facultad que se le antoje, anulará todos los demás poderes.

[...] El poder legislativo no debe tener la facultad de poner trabas al ejecutivo, porque la ejecución tiene sus límites en su naturaleza y es inútil limitarla: por otra parte, el poder ejecutor se ejerce siempre en cosas momentáneas. [...]
Lo que sí puede encontrarse en la obra de Montesquieu, como indica el epígrafe de la presente sección y el fragmento recién citado, es la separación de los poderes como una regla negativa que sí es compatible con la teoría dominante, la teoría de las funciones estatales (Troper, 1990:79). Esta regla negativa implica que debe ante todo evitarse confiar en un mismo órgano todas las funciones del Estado, en otras palabras, desconcentrar el poder.

Es importante aclarar que aunque existía un gran consenso en esta regla de no-concentración —al grado de ser propuesta por muchos más autores, no sólo Montesquieu-, no se habla de una forma particular de organizar el poder:

La regla no prescribe un modo particular de repartición del poder, se limita a prohibir una cierta organización. En principio era respetado siempre y cuando un mismo órgano no acumulase todos los poderes. Dicha acumulación es la definición misma del despotismo. Una vez que los poderes han sido repartidos, -se dice también $<<$ distribuidos $>>$, $<<$ divididos $>>0$ incluso $<<$ separados $>>-$ entre una multiplicidad de órganos, sea cual sea el modo de hacerlo, el despotismo es imposible. [...]

(El artículo de la Declaración de los Derechos del Hombre de 1789) fue aprobado sin debate mucho antes que los constituyentes hubieran podido lograr un compromiso sobre el tipo de Constitución que se adoptaría. Si se votó con tanta facilidad el artículo 16 es porque esta famosa separación de poderes no era una forma de gobierno particular.

[...] El artículo 16 simplemente expresa una verdad evidente: una Constitución no es otra cosa que una repartición de competencias. Una sociedad en la cual las competencias no son repartidas entre una multiplicidad de órganos, sino acumuladas en las manos de uno solo, no tiene Constitución. Esto, en otras palabras, significa que, visto que el despotismo es el cúmulo de poderes, una Constitución es precisamente el contrario del despotismo.

[...] < < Separar $>>$ en el idioma de la época no significaba como hoy establecer una frontera, sino simplemente distinguir, 0 incluso $<<$ repartir $>>$. (Troper, 1990:80-81). ${ }^{2}$

2 La traducción es propia. 
Así, si partimos de lo que los autores contemporáneos entienden por separación de poderes, designar el sistema de Montesquieu con este mismo nombre, como sostenía Eisenmannn (1933), no es probablemente lo más correcto. Lo que puede observarse en el Espíritu de las leyes entonces no es una separación funcional basada en el principio de especialización o material cuyo fundamento es la independencia, sino en una disposición tal que las autoridades no estén confundidas con las demás 0 incluso sean idénticas, y que no sean titulares de la totalidad de una función lo cual implicaría una necesaria cooperación de los distintos poderes para realizar una función. Se trata de lograr que realmente "el poder limite al poder" (Montesquieu, 2007: 144).

Evidentemente esta regla negativa, de origen teórico, debe ser complementada de tal forma que pueda tener una aplicación en la realidad. Es así como en el siglo XVIII comienza a hablarse de dos principios que ya se han mencionado a lo largo de esta sección, dos tipos de repartición del poder que posteriormente ayudarán a identificar entre distintos sistemas de gobierno: el principio de especialización y la independencia. El desarrollo de estos dos principios constituye dos de los sentidos de la locución de acuerdo con la teoría de Michel Troper.

\subsection{Especialización e independencia}

La doctrina del siglo XX identifica a la separación de poderes como un principio de técnica constitucional, un mecanismo que necesariamente debe estar presente, junto con otros tantos, en el diseño de un Estado constitucional para acotar exitosamente el poder. Este significado de separación de poderes se compone por dos principios que, como ya se mencionaban anteriormente, son la especialización y la independencia (Troper, 1990:74). ${ }^{3}$

La especialización implica que las tres funciones que clásicamente se le atribuyen al poder Estatal —la legislativa, la ejecutiva y la judicial- y se encuentran distribuidas entre distintos órganos, deben realizar sus respectivas facultades de modo exclusivo y enteramente - sin la coparticipación de otro- y como consecuencia natural no debe intervenir, impedir o privar de eficacia el ejercicio de las funciones de los otros.

La independencia, en cambio, atiende a las relaciones entre los órganos competentes para ejercitar las funciones estatales, los cuales deben ser suficientemente libres para actuar y eventualmente frenarse entre sí. Esto se refleja en el diseño de las distintas instituciones que dan vida a estos poderes, en especial su formación 0 nombramiento —el cual no debe ser realizado por otro órgano-, su funcionamiento y su duración - esto es, que su eventual revocación descanse en causas preestablecidas legalmente y no en la discrecionalidad de otros órganos-. Pero se encuentran en ocasiones también la prohibición de contacto físico entre los órganos, el establecimiento de independencia financiera e incluso de reglas sobre seguridad militar.

Vale la pena realizar un breve paréntesis para recordar que existen fuertes objeciones ${ }^{4}$ a esta doctrina que se han presentado a lo largo de los años y que han tenido que lidiarse no sólo desde una perspectiva teórica, sino práctica. En primer lugar, se señaló la contradicción con el principio de la indivisibilidad de la soberanía, conflicto cuya puerta de salida fue proporcionada por Alexander Hamilton en 1787, la cual consiste en considerar que el soberano no se manifiesta o expresa más que en la adopción o revisión de la Constitución y que, bajo la Constitución, nadie es soberano, cada órgano se encuentra esencialmente limitado (SaintBonnet, 2006:15). ${ }^{5}$

La segunda oposición se refiere a una inminente parálisis del Estado por la contraposición entre los poderes. Esta preocupación, que es específicamente

3 cfr. Del mismo autor, "La Ve République et la séparation des pouvoirs”, págs. 37-43; y Guastini, Riccardo, “¿Separación de los poderes o división del poder?", op. cit., pág. 304

4 Aquí sólo se abordarán y complementarán las críticas recordadas en Michel Troper, "Montesquieu e la separazione dei poteri negli Stati Uniti”.

5 La traducción es propia. 
de índole práctico, ha existido en todo momento y ha sido la motivación de una importante evolución institucional en la búsqueda de mejorar y hacer más eficiente el ejercicio de las funciones estatales. ${ }^{6}$

Una tercera crítica se refiere a la falsa percepción de equivalencia de las funciones, la no jerarquización de éstas y la consecuente imposibilidad de un real control interorgánico:

[...] Es incluso simplemente absurdo pretender que la actividad que consiste en hacer las leyes pueda ser equivalente a llevarlas a cabo. La segunda está evidentemente, por definición, subordinada a la primera. Pero si las funciones están jerarquizadas y los órganos especializados, se deduce que aquél que ejercita la función más elevada es superior a los otros. De acuerdo con la fórmula de Carré de Malberg, la jerarquía de los órganos sigue la jerarquía de las funciones, y un poder subordinado no podrá jamás frenar a un poder superior.

[...] Carré de Malberg tiene razón. No se puede alcanzar el equilibrio a través de la especialización y la independencia. Se dice entonces que se buscará 0 a través de la especialización 0 a través de la independencia, 0 a través de excepciones a una u otra [...]. (Troper, 1990:75,78)

A partir de este carácter cuasi-excluyente de los principios de independencia y especialización, se observará más adelante como algunos sistemas preferirán uno u otro principio, creando excepciones a la separación de poderes -en virtud de que la separación absoluta no sólo es inexistente, sino que sería inservible - en que generalmente se sacrificará el otro principio, aunque no necesariamente. Estas distintas interpretaciones de la separación de poderes, que serán exploradas en los siguientes apartados, generalmente se identifican como las interpretaciones europea y americana (Troper, 1990: 71-72).

\subsection{La separación funcional}

Referida también como la interpretación europea, la separación funcional es un sistema en el que las autoridades son solamente especializadas, pero no independientes. En este caso puede equipararse a la "separación de poderes" la locución "separación de funciones". Consiste, como se bosquejaba ya anteriormente, en una repartición de competencias en la que una autoridad ejercita una única función y no participa de forma decisiva al ejercicio de otra.

Este es el caso del sistema parlamentario, que abandona la independencia al surgir el gobierno del Parlamento, pero lleva a cabo la especialización, aunque existen excepciones. Lo importante es que la misma interdependencia orgánica permite la colaboración entre los mismos, aunque se trata de una colaboración desde su propio sector (Troper,1990:76). Es por esta cooperación de poderes que quienes apoyan esta interpretación la llaman "flexible".

Ante una posible contradicción en tanto que sí existe control entre los órganos —un ejemplo importante es la facultad del jefe de Estado de disolver el parlamento en un sistema parlamentario-, vale la pena recordar que en este contexto la independencia no se refiere a una forma de relación entre los órganos - si los actos de uno son susceptibles 0 no de revocación-, sino de un título de legitimidad -el no deber su dominación a otro órgano-: "La independencia proviene de la fuente de donde surge" (Troper, 2006:42)

\subsection{La separación material}

Se refiere a una necesaria independencia de los órganos,sinseréstosnecesariamenteespecializados. Es el caso de los regímenes presidenciales, por lo que también se conoce como la interpretación americana. Como se mencionaba anteriormente, el acento se encuentra en su designación y duración, y las excepciones se introducen principalmente a la especialización. Claros ejemplos de estas dos características son que, por un lado, en los regímenes presidenciales la independencia rigurosa impide la existencia de mecanismos de control propios de los sistemas parlamentarios como la 
disolución o la responsabilidad política del gobierno, y por el otro, que las autoridades no necesariamente se limitan a ejercer su función, como la participación del Presidente en la creación legislativa aparte de cumplir como órgano ejecutor.

En este punto, y a partir de haber analizado ya la separación funcional, nos cruzamos con importantes preguntas (Troper, 1990:76): ¿deberíamos decir que estamos en presencia de una separación rígida porque la independencia es rigurosa? ¿0 de una separación flexible? ¿Debe meterse el acento en el resultado previsto de las diversas excepciones y hablar de "checks and balances"? Y, de acuerdo con esta última hipótesis, ¿debe decirse que el sistema de "checks and balances" es sinónimo de "separación rígida" o de "separación flexible"?

A continuación se planteará un término que no pertenece a esta dicotomía, pero sirve como un paso más en la explicación teórica del fenómeno de separación de poderes y se acerca más a la teoría del control del poder.

\subsection{El equilibrio de poderes}

Identificada por Guastini como sinónimo de "división del poder" o "checks and balances", se trata de una técnica de organización constitucional en la que se busca que las autoridades, una vez separadas, se equilibren - la forma en la que esto se logra varía de sistema a sistema-. Pensada de esta forma, la separación de poderes es incompatible con el principio de especialización al no realizar los órganos sus funciones de forma exclusiva y en su totalidad; hay participación y las facultades suficientes para contraponerse a los otros, condicionando su ejercicio o frenándolo, logrando así que "el poder frene al poder". El objetivo de esto es evitar que los diversos órganos del Estado abusen de las competencias conferidas a ellos.

Como consecuencia, en primer lugar, el poder político, para evitar que se vuelva despótico, se distribuye 0 divide y confía a una pluralidad de órganos - 10 cual implica que, al ser necesaria la concurrencia, la acción de uno puede eventualmente ser impedida por la acción del otro-y posteriormente se otorgan poderes de control e influencia recíprocos (Guastini, 2000:305 \& Bryce, 1921:429). En un principio se consideró que el equilibrio no era concebible entre el poder legislativo y el ejecutivo, al no ser éstos poderes equivalentes ${ }^{7}$, por lo que únicamente se podría buscar dicho equilibrio en el seno del poder legislativo, dividiéndolo en dos cámaras que históricamente representaban estratos sociales distintos — como la Cámara de los Comunes y la Cámara de los Lores en Inglaterra - y que ahora se trata más de una representación de intereses diversos —un ejemplo, aunque no el único, es en el caso de los Estados federados en que se representa al pueblo por un lado y a los miembros de la federación en otra, como en Estados Unidos de América o México-. Para complementar este control se da, al igual que la competencia legislativa parcial, una competencia relativa a una función distinta (Troper, 1990:83). Posteriormente se fue dando cabida al ejecutivo recurriendo a diferentes técnicas, entre las que destaca el derecho de veto como parte del procedimiento legislativo en los sistemas presidenciales.

Otra aportación del primer sistema presidencial fue para evitar la potencial parálisis que un bloqueo entre poderes podía generar sin sacrificar su autonomía. Para lograr esto se condicionó la validez constitucional de ciertas actividades estatales consideradas como "puntos de contacto" o "puntos estratégicos del proceso político" a la necesaria cooperación de los distintos poderes, creando una interdependencia por coordinación - distinta a la interdependencia por integración de los sistemas parlamentarios - La aceptación generalizada de esta técnica convirtió a la exigencia de cooperación en un instrumento clave de la distribución y equilibrio de poderes (Loewenstein, 1976:133).

Este es el sistema que en el siglo XVIII se llamó "equilibrio de poderes", tesis que formó parte de

7 Esta afirmación ya fue mencionada. Véase la sección "Especialización e independencia” de este mismo capítulo. 
la cultura jurídica dominante en los tiempos de Montesquieu (Loewenstein, 1976:79), que se dice se realizó en Inglaterra y sirvió de inspiración a los constituyentes norteamericanos, quienes se vieron en la necesidad de realizar ciertas modificaciones para lograr adecuarlo a un gobierno republicano (p.83-85).

Esto parecería irónico, ya que la última sección describió este significado de "separación de poderes" como "un paso evolutivo en la teoría de la separación de poderes". Es importante tener en mente sin embargo que conceptos como "checks" y "balances" — o como suele traducirse al español, pesos o frenos y contrapesos - están ya presentes en la doctrina desde mucho tiempo atrás ${ }^{8}$, esto es, si bien nos referimos a esta interpretación como un "siguiente paso", no estamos hablando de una prelación o evolución de orden cronológico, sino conceptual. La existencia de trabajos en los que se hablaba ya de equilibrio entre poderes y cooperación no impidió en su momento el desarrollo de la concepción de la separación de poderes en que se debía elegir entre la especialización 0 la independencia -concepción que, aunque ha buscado demostrarse en estas líneas que tal vez no es la más adecuada para el estudio de los sistemas de gobierno, sigue utilizándose de manera considerable en nuestros días-.

\subsection{La separación vertical de poderes}

Si nos apegamos a la clasificación realizada por Michel Troper, esta separación se refiere específicamente a la repartición de competencias en sistemas federales (2006:34). Sin embargo, vale la pena realizar una breve revisión de la teoría de Karl Loewenstein que propone una definición del control vertical del poder e incorpora más elementos, aunque sólo se analizará a mayor profundidad el aspecto del federalismo.
En su obra, Loewenstein (1976) realiza una clasificación de los distintos tipos de controles de los Estados constitucionales. Por un lado se encuentran Ios controles horizontales, los cuales operan en el cuadro del aparato estatal y se mueven lateralmente al mismo nivel del aparato del dominio. Éstos deben ser completados y confrontados - señala el autor- con los controles verticales, los cuales son aquellos que implican una interacción -0 enfrentamiento - entre los detentadores del poder establecidos constitucionalmente y encargados de dirigir el proceso gubernamental —parlamento, gobierno, tribunales y electorado-, y la sociedad, la cual puede manifestarse de tres distintas formas, conformando así tres técnicas o campos de acción para este tipo de control: el federalismo; los derechos individuales y las garantías fundamentales; y el pluralismo.

El federalismo no se considera una técnica de control vertical sino hasta finales del siglo XVIII. Las primeras uniones de Estados de tipo federal se basaron en un principio en relaciones de vasallaje 0 feudales, por lo que se caracterizaron por la preponderancia de alguno de los miembros de la asociación; y/o una naturaleza más semiconfederal, reflejada en la ausencia de órganos comunes con jurisdicción directa sobre los ciudadanos de los Estados asociados. Es hasta la Constitución federal norteamericana de 1787 que los Estados americanos forman un Estado federal en el que puede hablarse de un verdadero control vertical, es decir, en el que hay un enfrentamiento entre dos soberanías estatales separadas territorialmente -dos grupos distintos de instituciones gubernamentales - y una repartición de competencias constitucionalmente establecida (Loewenstein, 1976:356). ${ }^{9} \quad$ Esta distribución de funciones permite la consecución de los fines nacionales y regionales al otorgar al Estado central y a los Estados miembros la autonomía

8 Véase, como un emblemático ejemplo la obra de Adams, John, "A Defense of the Constitutions of Government of the United States of America, Against the Attack of M. Turgot in His Letter to Dr. Price, Dated the Twenty-second Day of March, 1778", London, printed for John Stockdale, 1794/1974

9 Ningún estado federal puede funcionar sin una constitución escrita; ésta es la encarnación del contrato sobre la "alianza eterna". 
necesaria para funcionar independientemente de otro y a su vez permite la existencia de un equilibrio mutuo que responda a la necesidad de limitar el poder.

Aunque pueda pensarse al federalismo como una técnica constitucional diferenciada a la separación de poderes, la pertenencia a estos distintos sentidos de esta locución tiene sentido, como se mencionaba, ya que aplica el principio de la distribución del poder y consecuentemente es incompatible con un régimen autocrático —caracterizado por la concentración del poder, sea en un individuo, asamblea, comité, junta 0 partido-.

A partir de este breve análisis de los seis distintos sentidos de "separación de poderes" puede observarse una especie de continuidad entre los conceptos, en la que subyace la búsqueda de la desconcentración del poder. Sin embargo también se hallaron importantes diferencias, matices en cuyo fundamento se encuentra en la interpretación y aplicación de lo que inició como una regla negativa - los poderes no deben estar concentrados en un mismo órgano - que ha tenido un impacto significativo en el diseño institucional, y un tema que aún es objeto de debate en los actuales procesos de democratización.

En el caso de la separación funcional y material de los poderes, producto de un primer tipo de interpretación en el que se identificaron los principios de especialización e independencia, encontramos que es una teoría ampliamente difundida y que ha servido para la identificación de los distintos sistemas de gobierno: la separación funcional —donde se da preeminencia al principio de especializacióncomo característica de los sistemas parlamentarios y la separación material -en cuya base se encuentra el principio de independencia - propia de los sistemas presidenciales. Sin embargo, se trata de un conjunto teórico que ha traído consigo debates encaminados a determinar cuál es el mejor de los sistemas; la mejor forma de interpretar y aplicar esta particular forma de "separación de poderes"; cuáles son los elementos indispensables y cuáles son los prescindibles para poder aplicar exitosamente esta técnica constitucional en la realidad sin que pierda su esencia. Existe una tendencia a generalizar y estereotipar cuando se habla de rigidez (Troper, 1990:72) ${ }^{10}$ o flexibilidad de la separación de poderes en sistemas europeos comúnmente asociados con la idea de rigidez- y americanos -identificado como el más flexible-. Pero lo cierto es que se trata de una contraposición que no tiene realmente mucho sentido, al poder hablarse tanto de rigidez como de flexibilidad en cualquiera de los dos sistemas, ya que ambos han introducido excepciones -aunque distintas-. A esto se suma que hay un consenso teórico en este aspecto, y difícilmente se encuentra a algún autor que recomiende una separación rígida (p.73).

En ese sentido, parecería que una segunda interpretación de la separación de poderes en que hay una búsqueda de equilibrio entre los poderes - que implica una necesaria cooperación entre los mismos - es un paso adelante en el perfeccionamiento de la separación de los poderes que permite un control del poder más efectivo, en especial porque los métodos a los cuales se recurren para lograr este equilibrio varían de sistema a sistema.

Una observación que debe también tomarse en cuenta es que debemos distinguir entre la separación de poderes como producto teórico en la cual podemos hablar de paradigmas, realizar disecciones, clasificar, comparar ...- y como un producto histórico, fruto de la evolución de las instituciones como respuesta a cambios del entorno político-social e incluso económico:

Es interesante observar cómo en algunos de los países mencionados los pesos y contrapesos que existen -0 han existido - no fueron diseñados como mecanismos de seguridad, sino que fueron concomitantes con el proceso de transición de la monarquía u oligarquía a la democracia, los viejos poderes ejercitados por los Pocos cuya subsistencia 
fue permitida en una forma reducida junto con los nuevos poderes concedidos a los Muchos. Éste fue el origen de la Cámara de los Lores, que ahora actúa como Cámara Alta ${ }^{11}$. [...] También lo es la Segunda Cámara de Suecia, que sobrevive a las viejas Cuatro Cámaras de las Cuatro Órdenes (nobleza, clero, burguesía y campesinos) [...]. La mayoría de los Senados en los Estados modernos han sido concebidos como contrapeso de la Cámara Baja, siguiendo el ejemplo de la Antigua Roma o de Inglaterra. El veto del Presidente y del Gobernador de los Estados Unidos evoca el poder -ya en desuso desde mucho antes de 1787- que en algún momento perteneció a la Corona inglesa. Las restricciones a la acción legislativa contenidas en la Constitución norteamericana sí fueron nuevas, pero fueron surgiendo naturalmente durante la creación de Constituciones rígidas y junto con los arreglos de un sistema federal en el que se distribuyeron ciertos poderes al gobierno nacional dejando otros a los Estados (federados). (Bryce, 1921:434-435)

A partir de esta idea en que las instituciones obedecen al contexto y que no hay una fórmula específica para distribuir el poder, que ya había sido abordada en el capítulo anterior, además de la experiencia que se ha obtenido aplicando esta técnica y las dificultades que se han presentado, podemos hablar de un siguiente paso en la evolución de la conceptualización de la separación de poderes, el control del poder.

\section{EL CONTROL DEL PODER}

Si bien se conoce que el principio de separación de poderes indudablemente pertenece al "bagaje estándar" del Estado constitucional (Loewenstein, 1976:54), en los apartados anteriores se ilustraba ya cómo la concepción de la separación de poderes ha sido objeto de distintas interpretaciones. En este sentido, cuando las diversas obras que han servido de base para explicar en qué consiste el control del poder, hacen una crítica de la separación de poderes, es necesario advertir que se está pensando en un tipo específico de separación de poderes, a aquél en que debe elegirse entre los principios de especialización e independencia (Valadés, 2006:96). ${ }^{12}$ Una vez realizada esta aclaración, se procede a esbozar dichas críticas para entender la pertinencia de la teoría del control del poder como una alternativa para pensar los sistemas de gobierno.

Como sabemos, la separación de poder como exigencia del liberalismo político obedeció en su momento a un contexto determinado. Este esquema, de gran utilidad, sirvió de pauta para establecer la estructura de los gobiernos mediante la distinción y separación de los diversos campos de acción del Estado a través de los cuales se materializa el poder político. La delimitación en el ordenamiento jurídico de las esferas competenciales permitió también hablar de un principio de legalidad, en que la autoridad solamente puede ejercitar las funciones que le son expresamente atribuidas en el ordenamiento jurídico y que por lo tanto protege al gobernado de eventuales excesos en el ejercicio del poder al tratarse de actos, en caso de concretarse, que pueden ser nulos (Huerta, 2010: 15, 69-70 y 177-179).

Sin embargo, autores contemporáneos han calificado a la separación de poderes como un "dogma político" superado, alejado de la realidad (Loewenstein, 1976:54) y cuya aplicación lisa y llana incluso ha servido como un mecanismo de centralización del poder (Valadés, 2006:44). Limitarse a separar las funciones estatales resultó ser insuficiente, y la realidad lo demostró cuando los distintos sistemas dejaron de apegarse estrictamente a los principios de especialidad e independencia ${ }^{13}$ y comenzaron a

11 La traducción exacta sería Segunda Cámara, pero no se trata de una traducción convencional por lo que opté por utilizar el término que se utiliza en la doctrina, Cámara Alta. Lo mismo aplica para la Cámara Baja, cuya traducción literal sería Cámara popular.

En el caso de la Segunda Cámara de Suecia he respetado la literalidad de la traducción debido a la posterior referencia a las Cuatro Cámaras

12 En la obra de Valadés puede encontrarse esta observación cuando advierte los problemas que puede conllevar el uso del esquema reduccionista de la separación de poderes.

13 “[...]Incluso la más joven colección de constituciones después de la Segunda Guerra Mundial se mantiene firme en dicho principio (la separación de poderes), sin tener en cuenta su superación y alejamiento de la realidad en el siglo XX. Un simple vistazo 
introducir relaciones de control y cooperación entre los poderes mediante la realización conjunta simultánea o sucesiva - de las funciones estatales. Así, las constituciones contemplaban ya que los distintos órganos no ejercían una determinada actividad exclusivamente, sino primordialmente y se les sumaron otras funciones relativas a los otros, que material y estrictamente no les correspondían y que los convirtieron en órganos equilibradores no sólo entre sí, sino también dentro de sí y respecto del resto del sistema político (Huerta, 2010). ${ }^{14}$

Esta primera respuesta adaptativa se tradujo en un fenómeno de potenciación del poder (Valadés, 2006:1), el cual ha demostrado la facilidad con que pueden generarse asimetrías en las atribuciones y controles, produciendo potenciales "brotes de poder autoritario capaces de poner en riesgo la estructura constitucional del Estado democrático" (Valadés, 2006: 27, 114,133). ${ }^{15}$ Para evitar este riesgo se busca siempre, en caso de ser necesaria la atribución de más responsabilidades a un poder, que la potenciación sea bidireccional y lo más simétrica posible, es decir, que el poder de cada estructura constitucional debe ser, al menos en teoría, igual 0 equivalente en capacidad e influencia que los otros dos para mantener el equilibrio y evitar que un poder adquiera preponderancia respecto de los otros, así como tener las mismas posibilidades de efectuar actos que impliquen control del poder político ${ }^{16}$-a mayor poder, mayor control-.
El enorme dinamismo del contexto político —al cual se debe necesariamente agregar una creciente complejidad en virtud de la existencia de nuevos actores, como los partidos políticos ${ }^{17}$ y la generación de expectativas específicas respecto de cómo debe ser y ejercerse el poder en un sistema constitucional democrático (Valadés, 2006:144,147,158) - hace de las distintas concepciones de la separación del poder hasta ahora vistas como herramientas descriptivas y prescriptivas (Bobbio, 2007:9-10) insuficientes.

Es aquí donde un análisis de los controles como un auténtico sistema heterogéne $0^{18}$ y altamente dinámico cobra sentido. Considerar que los controles no actúan por sí solos -y por lo tanto no hay una única fórmula para controlar efectivamente al poder-, sino que son las distintas combinaciones de éstos y las interacciones resultantes lo que dotarán de sentido a un sistema constitucional (Valadés, 2006:16), y que deben contar con la posibilidad de responder a las cambiantes necesidades no sólo de sistema a sistema, sino incluso dentro de una misma experiencia constitucional con el paso del tiempo (Valadés, 2006:12,16,45), puede proporcionarnos una perspectiva más completa para entender los procesos del poder, diagnosticar puntual y oportunamente las deficiencias y proponer medidas más adecuadas para las dificultades que por la misma naturaleza del poder se irán seguramente presentando de manera constante.

\footnotetext{
a la forma más corriente de gobierno, el parlamentarismo, hubiera podido convencer a los autores constitucionales de que el poder legislativo y el ejecutivo no están separados ni personal ni funcionalmente." Loewenstein, Karl, op. cit., pág. 55

14 Una muy importante consecuencia del principio de legalidad que no debe dejar de recordarse es la certeza, es decir, la predictibilidad de las acciones del poder las hace confiables, existe una suposición fundada de lo que puede esperarse, las consecuencias de una determinada conducta (es posible esperar una cierta congruencia entre la conducta actual y la conducta futura) Cfr. Valadés, Diego, El control del poder, op. cit., pág. 23

15 Esto puede observarse en dos claros ejemplos de la historia estadounidense: la Gran Depresión y la Segunda Guerra Mundial. Estos eventos revelaron los límites de la elasticidad de los controles del sistema norteamericano.

16 Esto cobra aún más sentido si tomamos en consideración que el controlar al poder es en sí un acto de poder, una manifestación de poder. Así, el aumento de controles que acompaña a mayores atribuciones de un poder es una búsqueda de equilibrio y simetría en el poder.

cfr. Huerta Ochoa, Carla, op.cit., pág. 25 y Valadés, Diego, El control del poder, op.cit., págs. 17, 27 y 61

17 "Cuando Montesquieu escribía (El espíritu de las leyes) no existían las organizaciones políticas que hoy denominamos partidos, ni las organizaciones sociales que conocemos como sindicatos, ni los medios de comunicación tenían la presencia alcanzada en la actualidad, ni había emergido la modalidad bastante llamativa de las denominadas "organizaciones no gubernamentales", ni era de imaginar la aparición de nuevos órganos del poder no encuadrados en los tres tradicionales, como los ombudsman, o los organismos electorales autónomos, o los bancos centrales"

Valadés, Diego, El control del poder, op.cit., pág. 176
}

18 Manuel Aragón citado en Huerta Ochoa, Carla, op. cit., pág. 103 


\section{CONCEPTO DE CONTROL DEL PODER}

Parte de la ventaja que representa la perspectiva del control del poder, además de las razones expuestas en el apartado anterior, es que se considera a la separación relativa de funciones y la cooperación como un sistema de control básico (Huerta, 2010:17-18), el mecanismo con el que se inicia el control. Sin embargo, existe una clara convicción de la insuficiencia que la mera limitación de atribuciones estatales representa, y la consecuente necesidad de complementar estos mecanismos con la responsabilidad jurídica de los funcionarios para poder fiscalizar la actividad del poder y con mecanismos jurídicos que por un lado permitan prevenir abusos de poder y por el otro garanticen la efectividad de estas limitaciones. Esta idea se encontraba clara desde mucho tiempo atrás y se encontró presente en el proceso de creación de la Constitución norteamericana, como Thomas Jefferson lo señalaba claramente:

El despotismo electivo no fue el gobierno por el que nosotros luchamos; nosotros luchamos por un gobierno que no estuviese fundado sólo en los principios de la libertad, sino por uno en el que los poderes gubernamentales estuviesen de tal manera divididos y equilibrados entre las diferentes autoridades, que ningún poder pudiese traspasar sus límites legales sin ser eficazmente controlado y restringido por otros. (Loewenstein, 1976:131-132)

En ese orden de ideas propongo definir del control del poder (Huerta, 2010:112 \& Valadés, 2003:147,187) como un sistema heterogéneo y dinámico de instrumentos o mecanismos jurídicos y políticos establecidos en el ordenamiento jurídico con que cuentan los órganos constituidos para impedir, fiscalizar, responsabilizar y, en su caso, sancionar todo acto que se desvíe de las atribuciones constitucionales que les corresponden: todo esto con la finalidad de mantener el equilibrio de las relaciones entre los poderes sin afectar su efectividad.

La posterior evolución de los controles (Valadés, 2003:166-168) dejan diversas lecciones. Por una parte, incluso al hablar de Estados autocráticos, es posible ver que nunca ha existido un poder incontestado, aunque fuese sólo para legitimar su poder. Por otro lado, se puede también observar la constante actualización y el desarrollo de nuevas modalidades del control, entre los cuales destacan nuevas formas de control intraorgánicos que complementan a los controles interorgánicos, como la creación de contralorías —rama ejecutiva-, la adscripción de funciones públicas específicas a fracciones parlamentarias —rama legislativa - 0 la separación de la función profesional de juzgar y la tarea gubernativa de administrar los tribunales, encargada a los consejos de la judicatura 0 magistratura —rama judicial-.

Finalmente, se debe subrayar que la trascendencia del control del poder subyace el reconocimiento de que "Ia libertad es el telosideológico de la teoría de la separación de poderes" (Loewenstein, 1976:55,6372).$^{19}$ Esto es relevante debido a que asume la posibilidad de exigir responsabilidad política, esto es, la obligación de dar cuenta a otro detentador del poder sobre el cumplimiento de la función que le ha sido asignada, y la relevancia de la existencia de mecanismos que permiten la participación de minorías ${ }^{20}$ principalmente en funciones de control, una cuestión de gran importancia para la democracia (kelsen, 1988:341).

\section{CONCLUSIONES}

La naturaleza dinámica del poder político se ha manifestado a través de nuevos actores, escenarios, tensiones y necesidades que han fomentado interesantes cambios institucionales en un intento

19 La nueva división propuesta por Karl Loewenstein está compuesta por tres elementos: la decisión política conformadora o fundamental (policy determination), la ejecución de la decisión (policy execution) y finalmente el control político (policy control). Esta última es el concepto clave de la nueva división.

20 "Eso explica que Crisafulli (Stato..., p. 227) sustente que, en la actualidad, los dos "poderes" existentes son el de gobernar (que incluye la función normativa) y el de controlar. El primero concierne la mayoría; el segundo la minoría."

Valadés, Diego, El control del poder, op. cit., pág. 17 
de racionalizar todos los sistemas, incluyendo democracias ya consolidadas -esto es, ajustar 0 perfeccionar sus instituciones para que logren su propósito de una forma satisfactoria (Valdés,2003: 14)-. Estos cambios han retado a los significados más comunes del concepto de separación de poderes, que en muchas ocasiones han resultado ser insuficientes.

Pero estos debates no son tan novedosos como aparentan; la necesidad de ajustar la noción y las instituciones que dan vida a la separación de poderes ha existido desde mucho tiempo atrás, a pesar de que los contextos no eran tan complejos como ahora supuestamente lo son.

Este trabajo ha intentado demostrar este constante cambio basándose en la teoría de la polisemia de Michel Troper, que analiza los diferentes sentidos del concepto que con el tiempo han aparecido, y que de alguna manera su evolución como una respuesta las necesidades que fueron presentándose en la traducción de la teoría a la práctica.

El propósito de este artículo no ha sido meramente recuperar esta interesante teoría, sino también realizar una contribución al subrayar la naturaleza dinámica del poder político y por lo tanto proponiendo la teoría del control del poder como una alternativa útil 0 un "nuevo paso evolutivo" del concepto de separación de poderes. El control del poder, al ser concebido como un sistema heterogéneo que asume la necesidad de flexibilizar, resulta una opción pertinente con la apertura suficiente para admitir variaciones que le permitan adaptarse a distintos contextos e incluso a los cambios que se presenten con el paso del tiempo en un mismo sistema, siempre considerando que la finalidad de cualquier diseño constitucional es la limitación del poder y la preservación de la libertad.

La realidad ha probado que el debate en torno a la separación de poderes es un debate vivo, y la prueba está en que se han realizado cambios significativos en el diseño institucional de los sistemas constitucionales contemporáneos, por ejemplo, la adopción de instituciones parlamentarias en regímenes presidenciales y viceversa, o la creación de agencias de gobierno autónomas -organismos cuyos mecanismos de nombramiento, operación y financiamiento garantizan su independencia frente a los tres poderes clásicos del Estado-, y que prometen tener un papel protagónico en los procesos de transición o perfección de la democracia.

\section{REFERENCIAS BIBLIOGRÁFICAS}

- Adams, J. (1778). A Defense of the Constitutions of Government of the United States of America, Against the Attack of M. Turgot in His Letter to Dr. Price, Dated the Twenty-second Day of March. London: printed for John Stockdale, 1794/1974

- Bobbio, N. (2007). La teoría de las formas de gobierno en la historia del pensamiento político traducción de José F. Fernández Santillán, 2a ed., México: Fondo de Cultura Económica, colección Política y Derecho.

- Bryce, J. (1921). Modern Democracies. Vol. II. London: MacMillan and Co. Limited.

- Eisenmann, Charles, (1933). "L' "Esprit des lois" et la séparation des pouvoirs", in R. Crarré de Malberg, Mélanges, Paris: Librairie du Recueil Sirey (société anonyme).

- Guastini, R. (2000). ¿Separación de los poderes o división del poder?, in Revista de la Facultad de Derecho de México, México, tomo L, números 229-234, pp. 299-319

- Hans, K. (1988). Teoría general del derecho y del Estado, 4a ed., México: UNAM.

- Huerta, C. (2010). Mecanismos Constitucionales para el Control del Poder Político, $3^{\mathrm{a}}$ ed. México: Universidad Nacional Autónoma de México.

- Loewenstein, K, (1976). Teoría de la constitución, 2a ed. Barcelona: Editorial Ariel.

- Montesquieu. (2007). El espíritu de las leyes, 17a ed., versión castellana de Nicolás Estévanez, México: Porrúa, colección "Sepan cuántos..." núm. 191.

- Saint-Bonnet, F. (2006). Le constitutionnalisme libéral français en trompe-oeil. Actualité de l'autre Montesquieu, in Droits. Revue française de théorie, de philosophie et de culture 
juridiques. La Ve République: des institutions liberales?, Paris.

- Salazar, P. (2006.) La democracia constitucional. Una radiografía teórica, México: Fondo de Cultura Económica, Instituto de Investigaciones Jurídicas de la UNAM, Política y derecho.

- Troper, M, (1990). Montesquieu e la separazione dei poteri negli Stati Uniti", in Materiali per una Storia della Cultura giuridica. Genova, Anno XX, num. 1, giugno

- Troper, M. (2006). La Ve République et la séparation des pouvoirs, in Droits. Revue française de théorie, de philosophie et de culture juridiques. La Ve République: des institutions liberales?, Paris

- Valadés, D. (2003). La parlamentarización de los sistemas presidenciales. México: Universidad Nacional Autónoma de México, Instituto de Investigaciones Jurídicas, Serie Estudios Jurídicos.

- Valadés, D. (2006). El control del poder, 3a ed. México: Universidad Nacional Autónoma de México, Editorial Porrúa. 